\title{
Survival outcomes in pediatric recurrent high-grade glioma: results of a 20 -year systematic review and meta-analysis
}

\author{
Cassie Kline $^{1}$, Erin Felton ${ }^{2}$, I. Elaine Allen ${ }^{3}$, Peggy Tahir ${ }^{4}$, and Sabine Mueller ${ }^{1,2,5}$ \\ ${ }^{1}$ Division of Hematology/Oncology, Department of Pediatrics, University of California, San \\ Francisco, $55016^{\text {th }}$ Street, $4^{\text {th }}$ Floor, San Francisco, CA 94158 \\ 2Department of Neurology, University of California, San Francisco, 550 Sandler Neurosciences, \\ 625 Nelson Rising Lane, 402B, Box 0434, San Francisco, CA 94158 \\ ${ }^{3}$ Department of Epidemiology \& Biostatistics, University of California, San Francisco, $55016^{\text {th }}$ \\ Street, $2^{\text {nd }}$ Floor, San Francisco, CA 94158 \\ ${ }^{4}$ UCSF Library, University of California, San Francisco, 530 Parnassus Avenue, San Francisco, \\ CA 94143 \\ ${ }^{5}$ Department of Neurological Surgery, University of California, San Francisco, 505 Parnassus \\ Avenue, M779, San Francisco, CA 94143
}

\begin{abstract}
Recurrent pediatric high-grade glioma is a leading cause of cancer-related death in children. We report results of a systematic review and meta-analysis investigating survival outcome in pediatric patients with recurrent high-grade glioma over the last 20 years.

Methods-MEDLINE/PubMed, EMBASE, Web of Science and Cochrane Review databases were searched for relevant studies reporting on survival outcomes for pediatric patients with recurrent high-grade glioma treated between 1996-2016. Progression-free survival (PFS) and overall survival (OS) were calculated cumulatively over all studies, by therapy subgroup, and by decade of treatment. Random effects models were used to control for heterogeneity as measured by the $\mathrm{I}^{2}$ statistic.
\end{abstract}

Results-A total of 17 studies across 4 treatment strategies were included. Eleven investigated traditional chemotherapy, 1 investigated targeted therapy, 3 investigated immunotherapy, and 2 investigated radiotherapy. A total of 129 patients were included with a median age of 10.0 years. Cumulative PFS was 3.5 months (95\% CI 2.1, 5.0). Cumulative OS was 5.6 months (95\% CI 3.9, 7.3). OS was 4.0 months $(95 \%$ CI 1.9, 6.1) using traditional chemotherapy, 9.3 months using targeted therapies (95\% CI 5.4, 13), 6.9 months using immunotherapy (95\% CI 2.1, 12), and 14

Corresponding author: Cassie Kline, MD, MAS, Division of Pediatric Hematology/Oncology; Department of Pediatrics, University of California, San Francisco, $55016^{\text {th }}$ Street, $4^{\text {th }}$ Floor, San Francisco, CA 94158, Phone (415) 476-3831; Fax (415) 353-2657, cassie.kline@ucsf.edu.

Conflict of Interest: No contributing authors have any conflict of interest to declare.

This work was presented as a podium presentation at the $4^{\text {th }}$ Pediatric Neuro-Oncology Basic and Translational Research Conference in New York, NY on June 16, 2017 and as an eTalk at the Society of Neuro-Oncology Annual Meeting on November 17, 2017. 
months using reirradiation (95\% CI 2.8, 25). OS between 1996 and 2006 was 4.2 months (95\% CI 2.1, 6.2) compared to 8.5 months $(95 \%$ CI 5.6, 11) after 2006.

Conclusions-Pediatric patients with recurrent high-grade glioma suffer from poor PFS and OS, regardless of therapy. There may be a trend towards improved OS in the last decade.

\section{Keywords}

Pediatric high-grade glioma; recurrence; survival; outcomes

\section{Introduction}

Recurrent pediatric high-grade glioma has no known cure, despite years of clinical trials testing new therapeutic approaches. High-grade gliomas are comprised of mixed astrocytic tumors; anaplastic astrocytomas and oligodendrogliomas, WHO grade III; and glioblastomas, WHO grade IV.[1] Up-front therapy for these tumors typically includes surgery with the goal of gross total resection, radiation, and chemotherapy. Estimates on overall survival (OS) for newly diagnosed pediatric high-grade glioma vary according to anatomic location such as supratentorial versus the brainstem versus the spinal cord. For tumors located supratentorially, OS is less than $20 \%$ at 5-years, with most patients dying within two years of diagnosis. [2,3] Treatment options are more limited in the recurrent setting and are frequently based on prior therapy exposure. For these patients, there is no standard of care therapy and enrollment on a clinical trial is frequently pursued. These trial options may offer intravenous chemotherapy, oral agents, immune-based therapy, or repeat irradiation.[4] Most trials in the recurrent high-grade glioma setting are single-arm and report progression-free survival (PFS) and/or OS; however, there remains limited aggregate data on survival outcomes for this group. This presents limitations when designing trials as statistical design, including effect size and power calculations, is frequently based on anecdotal evidence or on data from the most recently published clinical trial. This current systematic review and meta-analysis presents a comprehensive review on the outcomes of recurrent pediatric high-grade glioma, excluding gliomas of the brainstem and spinal cord, from a variety of treatment options spanning the last twenty years.

The aims of our study are to provide a resource of survival outcomes for this high-risk population. Such findings will provide insight into clinical trial design including development of appropriate effect sizes and power calculations. The results will also offer providers, patients, and families more accurate expectations of survival for this population.

\section{Materials and Methods}

\section{Search Strategy}

A systematic literature search was conducted using MEDLINE, Embase, Cochrane Library, and Web of Science. The search string was comprised of the following MESH terms and Booleans: ("Astrocytoma/anatomy and histology"[Mesh] OR "Astrocytoma/genetics"[Mesh] OR "Astrocytoma/mortality"[Mesh] OR "Astrocytoma/statistics and numerical data"[Mesh] OR "Astrocytoma/therapy"[Mesh] OR "Glioma/anatomy and histology"[Mesh] OR "Glioma/genetics"[Mesh] OR "Glioma/mortality"[Mesh] OR "Glioma/statistics and 
numerical data"[Mesh] OR "Glioma/therapy"[Mesh] OR "Glioblastoma/anatomy and histology"[Mesh] OR "Glioblastoma/genetics"[Mesh] OR "Glioblastoma/mortality"[Mesh] OR "Glioblastoma/statistics and numerical data"[Mesh] OR "Glioblastoma/therapy"[Mesh]) AND ("recurrent disease"[All Fields] OR ("recurrence"[MeSH Terms] OR "recurrence"[All Fields]) OR recurrent[All Fields]) AND ((Case Reports[ptyp] OR Clinical Study[ptyp] OR Clinical Trial[ptyp] OR Comparative Study[ptyp] OR Controlled Clinical Trial[ptyp] OR Evaluation Studies[ptyp] OR Multicenter Study[ptyp] OR Observational Study[ptyp] OR Randomized Controlled Trial[ptyp]) AND ("1996/01/01"[PDAT] : "2016/12/31"[PDAT]) AND "humans"[MeSH Terms] AND English[lang] AND (("infant"[MeSH Terms] OR "child"[MeSH Terms] OR "adolescent"[MeSH Terms]) OR "young adult"[MeSH Terms])).

Non-MESH search terms and hand searching of the literature were also done for search completeness. The search period was from January 1, 1996 to December 31, 2016. Only studies published in the English language were included. The resultant studies were individually reviewed for inclusion in the final analysis and as based on information within the title, abstract, and/or main text (CK). Adjudication occurred by independent review if necessary (EF, SM). The study was registered on PROSPERO (CRD 42016050359) prior to initiation of data collection and investigators followed PRISMA guidelines for systematic reviews and meta-analyses.[5]

\section{Selection criteria}

Inclusion criteria for the studies was as follows: 1) Histologically confirmed high-grade glioma (biopsy at initial diagnosis sufficient, including any WHO grade III or IV tumors) excluding brainstem or spinal cord tumors as well as malignant gliomas arising from prior low-grade glioma or as secondary malignancy; 2) Patient age < 25 years old at time of recurrence; 3) Publication year between January 1, 1996 and December 31, 2016; 4) Study types including clinical trials, cohort studies, or case series with at least 5 patients; 5) Reported outcome including PFS and/or OS; and 6) Publication written or translated in the English language.

\section{Data extraction}

The data extracted from each study included authors, year of publication, study design, number of patients included, age of patients, disease pathology, study intervention, and reported PFS and/or OS. For studies including patients with age range of less than and greater than 25 years, data on individual patients less than 25 years were reviewed and extracted, as available. If individual patients within the targeted age range and meeting all other inclusion criteria were able to be extracted, these patients were individually identified and included in the final statistical analysis.

\section{Statistical analysis}

A random effects model (DerSimonian-Laird method) was used for all analyses. Because it was not possible to assume that all studies came from the same population and followed the same protocol, random effects models were used to control for heterogeneity between study as measured by the $\mathrm{I}^{2}$ statistic. PFS and OS with $95 \%$ confidence intervals were calculated cumulatively over all studies, by therapy subgroup, and by decade of treatment (i.e. January 
1, 1996 to December 31, 2006 and January 1, 2007 to December 31, 2016). Therapy subgroups included traditional chemotherapy, targeted therapy, immunotherapy, and radiation therapy. All p-values were calculated using a two-sided test. A p-value $<0.05$ was considered statistically significant. All statistical analyses were done using Stata14.0 (StataCorp LP, College Station, TX). Study bias was assessed per individual study, looking for area of bias such as selection bias, performance bias, attrition bias, and reporting bias. Bias was further assessed using the Egger test and visually using funnel plots plotting sample size versus effect size.

\section{Results}

\section{Study characteristics}

The systematic literature search was completed on April 19, 2017. A total of 2062 articles were initially identified from MEDLINE ( $n=874)$, Embase ( $n=583$ ), Cochrane Library $(n=551)$ and Web of Science $(n=54)$. A total of 569 articles were excluded due to duplication. A total of 1467 articles were excluded due to age of patients outside of inclusion range $(n=45)$, diagnoses other than high-grade glioma $(n=641)$, newly diagnosed disease ( $n=69)$, incorrect publication type $(n=209)$, wrong outcome reported $(n=386)$, year outside of inclusion ranges $(n=4)$, publication language other than English $(n=9)$, and inability to determine age or diagnoses of patient population $(n=104)$. An additional 9 studies were excluded based on final patient number being 1 and/or missing standard deviation (figure 1). A total of 17 articles were included for the final review[6-23] - 11 using traditional chemotherapy, 1 using targeted therapy, 3 using immunotherapy, and 2 using radiation therapy (supplemental table 1). There were two retrospective analyses, the remaining were all prospective, including phase 1 and 2 single or multi-center investigations. All studies investigated only single-arm therapy options and no study included randomization. Out of 17 studies included in the final analysis, only 1 offered aggregate data that met all inclusion/exclusion criteria. The remaining 16 studies required isolation of data per individual patient. A total of 129 patients were included across the final 17 studies with a median age of 10.0 years of all patients.

\section{Heterogeneity and Bias}

There was no heterogeneity across studies as indicated by $\mathrm{I}^{2}$ statistics of $0.0 \%$ for both PFS and OS meta-analyses ( $\mathrm{p}$-value $=1.00$-PFS; $\mathrm{p}$-value $=0.796-\mathrm{OS}$ ). Assessment of bias in individual studies was limited due to the lack of randomized control studies included in the final analyses. Funnel plots were visually inspected to assess for bias. The PFS funnel plot demonstrated some deviation away from the median towards shorter PFS. For OS, there was equal distribution across all sample sizes. We also evaluated publication bias using Egger's test on OS and PFS and they were not significant. No additional studies were excluded for bias.

\section{Survival analyses}

Eleven studies included measurement of PFS (figure 2). Pooled PFS was 3.5 months (95\% CI 2.1, 5.0; $\mathrm{p}$-value $<0.001 ; \mathrm{I}^{2}=0.0 \%$ ). Sixteen studies included measurement of OS (figure 3). Pooled OS was 5.6 months (95\% CI 3.9, 7.3; p-value $<0.001 ; \mathrm{I}^{2}=0.0 \%$ ). 
Across therapy subgroups, PFS was 3.5 months (95\% CI 1.9, 5.0; p-value < 0.001 ) for traditional chemotherapy, 4.4 months for targeted therapy $(95 \% \mathrm{CI}-15,24 ; \mathrm{p}$-value $=$ 0.653 ), and 4.1 months for radiotherapy (95\% CI 0.2, 8.0; p-value $=0.041$; Supplemental figure 1). OS was 4.0 months (95\% CI 1.9, 6.1; p-value < 0.001) for traditional chemotherapy, 9.3 months for targeted therapy (95\% CI 5.4, 13; p-value < 0.001 ), 6.9 months $(95 \%$ CI 2.1, 12; p-value $=0.005)$ for immunotherapy, and 14 months for radiotherapy $(95 \%$ CI $2.8,25$; p-value $=0.015$; figure 4$)$.

Across decade subgroups, PFS was 3.6 months (95\% CI 2.1, 5.0; p-value < 0.001$)$ for the first decade (1996-2006) and 3.3 months $(95 \%$ CI $-2.6,9.2$; p-value $=0.269)$ for the second decade (2007-2016; Supplemental figure 2). OS was 4.2 months (95\% CI 2.1, 6.2; p-value < $0.001)$ for the first decade and 8.5 months $(95 \%$ CI 5.6, 11; p-value < 0.001$)$ for the second decade (figure 5).

\section{Discussion}

The outcomes in pediatric high-grade glioma remain dismal especially at time of recurrence. Standard therapy for newly diagnosed pediatric high-grade glioma involves a multi-modal approach, while at time of recurrence, enrollment on clinical trials is frequently pursued. Design and outcome hypotheses of clinical trials for pediatric high-grade glioma frequently rely on historical controls, as based on the most recently published clinical trial and due to limited comprehensive survival data.[24-26] Additionally, given the lack of publications on cumulative data, it can be challenging for providers to offer estimated survival for this population. Aggregate outcome data over time can offer a resource for outcomes in recurrent pediatric high-grade glioma and lead to more accurate clinical trial design in regards to effect sizes as well as improve anticipatory guidance for patients and families. This study aims to offer insight into these issues and provide comprehensive outcome data for recurrent pediatric high-grade glioma from publications over the last two decades.

Our findings confirm the devastating prognoses recurrent high-grade glioma carries in children. In our investigation, the median PFS for recurrent high-grade glioma is only 3.5 months and median OS is only 5.6 months. These results occur across a variety of therapeutic approaches, ranging from more traditional high-dose chemotherapy to targeted treatments to reirradiation. In subgroup analyses, the trend towards prolonged OS in the last ten years may be promising that advances are being made in treating this disease. This would be supported by evidence of longer PFS and OS with the use of more recently developed therapies such as immunotherapy and targeted agents. Ideally, both treatment strategies will prove to hold less systemic toxicity than traditional therapies. Reirradiation has previously been shown to be feasible and offer potential survival benefit in children with recurrent high-grade glioma and this was at least somewhat supported by our findings, where one study showed reirradiation offered an OS of 11.4 months.[13, 27]

We anticipated heterogeneity would be present in our review due to the large range of years included, variable study designs and interventions, and a broad patient age range. Heterogeneity was not indicated by the $\mathrm{I}^{2}$ statistic for each analysis; however, it should be noted that the $\mathrm{I}^{2}$ statistic in our review may be limited by the small number of studies in our 
final analyses. Regardless, in depth review of all studies revealed that the patient populations appear consistently similar across studies. Studies showing both the longest and shortest survivals appear to span similar ages, equal distributions among sex, comparable inclusion of both grade 3 and grade 4 high-grade glioma, and equivalent prior therapy exposures. For example, the studies reporting the longest and shortest OS each included 50\% males with respective median ages of 9.5 years and 10 years, a mix of AA and GBM, and a variety of patients previously treated with surgery, radiation, and chemotherapy, or combinations thereof. Such similar patient populations across the studies makes it more likely the spread across PFS and OS is due to therapeutic intervention as opposed to intrinsic differences in patient population and study heterogeneity. Additionally, the evidence of prolonged OS with more recently developed therapies such as targeted approaches and immunotherapy correlates with increased OS in the last ten years. Findings should still be interpreted with caution given the small number of studies and with attention being paid to the fact that only one study meeting our selection criteria applied targeted therapy and one applied external beam radiation.

There are potential limitations within our study. Assessment of bias within the individual studies included in this review is challenging as no study followed a randomized controlled design. All studies were single-arm investigations looking at objective survival outcomes. Given the objective nature of the outcome measures and the propensity for many of the studies to individually detail each patient outcome without censorship or exclusion of patient outcome data, there does appear to be some protection against intrinsic study bias. Another potential limitation of extrapolating progression outcomes and comparing across multiple studies may arise from differing definitions of progression across studies. For instance, some studies did not clearly outline what they defined as progression, some used progression by a certain percentile in any disease parameter, and other applied progression as based on the sum of tumor dimensions. Regardless, we still find the cumulative survival outcomes to be pertinent information for clinical trial development. Additionally, our review includes only one immunotherapy-based study reporting on PFS and only one targeted therapy study reporting on OS. Thus, the data reported for these subgroups are limited and subject to bias. Though, the patient population characteristics of these studies were similar to others and the PFS for the immunotherapy was within the range for the other investigated studies making bias within these studies seem less likely. Another shortcoming of our report is that all included immunotherapy-based protocols centered on the use of dendritic cell vaccines. Such few studies in each treatment category may limit the application of the outcomes in our subgroups to broader, all-inclusive targeted and immunotherapies. Four studies in our review had one to two long-term survivors that were censored at time of study completion.[8, 18, $22,28]$ However, by using median survival, our results should not be impacted by this censorship. Our PFS funnel plot was also skewed towards shorter PFS, potentially indicating publication bias; however, given the deviation occurred across multiple studies of all sample sizes and is skewed towards null findings, it is unlikely the funnel plot reflects publication bias. Lastly, the studies included in the final analyses had small sample sizes with an average of only 8 patients per study. We feel these small size studies though speak to the rarity of this disease as opposed to the quality of studies. 
Even with improved OS across recent therapies, our study confirms that regardless of the therapeutic intervention employed, PFS and OS for pediatric recurrent high-grade glioma is unlikely to span more than a few months. This calls for continued development of novel therapy approaches. We propose development of clinical trials to support investigational therapies should rely on aggregate outcome data such as that reported in our meta-analysis. By using more comprehensive data, power and effect size calculations will be more reflective of appropriate anticipated outcomes. This may prevent premature closure of trials as based on outcomes that are falsely assumed to be inferior. Additionally, providers, patients, and families can benefit from cumulative outcome data to provide appropriate expectations on patient survival.

\section{Conclusion}

Children with recurrent high-grade glioma exhibit extremely poor outcomes and thus deserve investigation of innovative treatments. Our findings can serve as the basis for accurate design of such trials and offer families and providers appropriate anticipatory guidance of survival for this patient population.

\section{Supplementary Material}

Refer to Web version on PubMed Central for supplementary material.

\section{Acknowledgments}

Funding: No external funding was used to directly support this study.

C.N.K. is supported by the National Institutes of Health T32 grant (CA128583), UCSF Clinical and Translational Science Institute Strategic Opportunities Support Program (A119683), Alex's Lemonade Stand Foundation (A120729), and Frank A. Campini Foundation. S.M. is supported by the National Institutes of Health National Center for Advancing Translational Sciences through UCSF-CTSI (KL2TR000143).

\section{References}

1. Cage TA, Mueller S, Haas-Kogan D, Gupta N. High-grade gliomas in children. Neurosurg Clin N Am. 2012; 23:515-523. DOI: 10.1016/j.nec.2012.04.007 [PubMed: 22748663]

2. Broniscer A, Gajjar A. Supratentorial high-grade astrocytoma and diffuse brainstem glioma: two challenges for the pediatric oncologist. The oncologist. 2004; 9:197-206. [PubMed: 15047924]

3. Gottardo NG, Gajjar A. Chemotherapy for malignant brain tumors of childhood. Journal of child neurology. 2008; 23:1149-1159. DOI: 10.1177/0883073808321765 [PubMed: 18952581]

4. Braunstein S, Raleigh D, Bindra R, Mueller S, Haas-Kogan D. Pediatric high-grade glioma: current molecular landscape and therapeutic approaches. Journal of neuro-oncology. 2017; doi: 10.1007/ s11060-017-2393-0

5. Liberati A, Altman DG, Tetzlaff J, Mulrow C, Gotzsche PC, Ioannidis JP, Clarke M, Devereaux PJ, Kleijnen J, Moher D. The PRISMA statement for reporting systematic reviews and meta-analyses of studies that evaluate health care interventions: explanation and elaboration. J Clin Epidemiol. 2009; 62:e1-34. DOI: 10.1016/j.jclinepi.2009.06.006 [PubMed: 19631507]

6. Mahoney DH Jr, Strother D, Camitta B, Bowen T, Ghim T, Pick T, Wall D, Yu L, Shuster JJ, Friedman H. High-dose melphalan and cyclophosphamide with autologous bone marrow rescue for recurrent/progressive malignant brain tumors in children: a pilot pediatric oncology group study. $\mathrm{J}$ Clin Oncol. 1996; 14:382-388. DOI: 10.1200/JCO.1996.14.2.382 [PubMed: 8636747]

7. Chamberlain MC. Recurrent supratentorial malignant gliomas in children. Long-term salvage therapy with oral etoposide. Archives of neurology. 1997; 54:554-558. [PubMed: 9152112] 
8. Wagner S, Erdlenbruch B, Langler A, Gnekow A, Kuhl J, Albani M, Volpel S, Bucsky P, Emser A, Peters O, Wolff JE. Oral topotecan in children with recurrent or progressive high-grade glioma: a Phase I/II study by the German Society for Pediatric Oncology and Hematology. Cancer. 2004; 100:1750-1757. DOI: 10.1002/cncr.20168 [PubMed: 15073866]

9. Shih CS, Hale GA, Gronewold L, Tong X, Laningham FH, Gilger EA, Srivastava DK, Kun LE, Gajjar A, Fouladi M. High-dose chemotherapy with autologous stem cell rescue for children with recurrent malignant brain tumors. Cancer. 2008; 112:1345-1353. DOI: 10.1002/cncr.23305 [PubMed: 18224664]

10. Saurez G, Cabanas R, Zaldivar M, Garnier T, Iglesias B, Piedra P, Castillo MR, Longchong M, Iznaga N, Lage A. Clinical experience with nimotuzumab in cuban pediatric patients with brain tumors, 2005 to 2007. MEDICC Rev. 2009; 11:27-33. [PubMed: 21483304]

11. Parekh C, Jubran R, Erdreich-Epstein A, Panigrahy A, Bluml S, Finlay J, Dhall G. Treatment of children with recurrent high grade gliomas with a bevacizumab containing regimen. Journal of neuro-oncology. 2011; 103:673-680. DOI: 10.1007/s11060-010-0444-x [PubMed: 21038110]

12. Lasky JL 3rd, Panosyan EH, Plant A, Davidson T, Yong WH, Prins RM, Liau LM, Moore TB. Autologous tumor lysate-pulsed dendritic cell immunotherapy for pediatric patients with newly diagnosed or recurrent high-grade gliomas. Anticancer Res. 2013; 33:2047-2056. [PubMed: 23645755]

13. Muller K, Scheithauer H, Pietschmann S, Hoffmann M, Rossler J, Graf N, Baumert BG, Christiansen H, Kortmann RD, Kramm CM, von Bueren AO. Reirradiation as part of a salvage treatment approach for progressive non-pontine pediatric high-grade gliomas: preliminary experiences from the German HIT-HGG study group. Radiat Oncol. 2014; 9:177.doi: 10.1186/1748-717X-9-177 [PubMed: 25112658]

14. Ardon H, De Vleeschouwer S, Van Calenbergh F, Claes L, Kramm CM, Rutkowski S, Wolff JEA, Van Gool SW. Adjuvant dendritic cell-based tumour vaccination for children with malignant brain tumours. Pediatric Blood and Cancer. 2010; 54:519-525. DOI: 10.1002/pbc.22319 [PubMed: 19852061]

15. Bouffet E, Khelfaoui F, Philip I, Biron P, Brunat-Mentigny M, Philip T. High-dose carmustine for high-grade gliomas in childhood. Cancer Chemother Pharmacol. 1997; 39:376-379. DOI: 10.1007/s002800050586 [PubMed: 9025780]

16. Bouffet E, Mottolese C, Jouvet A, Philip I, Frappaz D, Carrie C, Brunat-Mentigny M. Etoposide and thiotepa followed by ABMT (autologous bone marrow transplantation) in children and young adults with high-grade gliomas. European journal of cancer. 1997; 33:91-95. [PubMed: 9071906]

17. De Sio L, Milano GM, Castellano A, Jenkner A, Fidani P, Dominici C, Donfrancesco A. Temozolomide in resistant or relapsed pediatric solid tumors. Pediatric Blood and Cancer. 2006; 47:30-36. DOI: 10.1002/pbc.20516 [PubMed: 16047361]

18. Gilheeney SW, Khakoo Y, Souweidane M, Wolden S, Boulad F, Dunkel IJ. Thiotepa/topotecan/ carboplatin with autologous stem cell rescue in recurrent/refractory/poor prognosis pediatric malignancies of the central nervous system. Pediatric Blood and Cancer. 2010; 54:591-595. DOI: 10.1002/pbc.22347 [PubMed: 19998470]

19. Gilman AL, Jacobsen C, Bunin N, Levine J, Goldman F, Bendel A, Joyce M, Anderson P, Rozans M, Wall DA, Macdonald TJ, Simon S, Kadota RP. Phase I study of tandem high-dose chemotherapy with autologous peripheral blood stem cell rescue for children with recurrent brain tumors: A pediatric blood and marrow transplant consortium study. Pediatric Blood and Cancer. 2011; 57:506-513. DOI: 10.1002/pbc.22899 [PubMed: 21744474]

20. Iwami K, Shimato S, Ohno M, Okada H, Nakahara N, Sato Y, Yoshida J, Suzuki S, Nishikawa H, Shiku H, Natsume A, Wakabayashi T. Peptide-pulsed dendritic cell vaccination targeting interleukin-13 receptor alpha2 chain in recurrent malignant glioma patients with HLA-A*24/A*02 allele. Cytotherapy. 2012; 14:733-742. DOI: 10.3109/14653249.2012.666633 [PubMed: 22424217]

21. Lashford LS, Thiesse P, Jouvet A, Jaspan T, Couanet D, Griffiths PD, Doz F, Ironside J, Robson K, Hobson R, Dugan M, Pearson ADJ, Vassal G, Frappaz D. Temozolomide in malignant gliomas of childhood: A United Kingdom Children's Cancer Study Group and French Society for Pediatric Oncology Intergroup Study. Journal of Clinical Oncology. 2002; 20:4684-4691. DOI: 10.1200/ JCO.2002.08.141 [PubMed: 12488414] 
22. Papadakis V, Dunkel IJ, Cramer LD, Kramer E, Papadopoulos E, Goldman S, Packer RJ, Willoughby M, Baker D, Garvin J, Strandjord S, Coccia P, Kaplan AM, Klemperer M, Finlay JL. High-dose carmustine, thiotepa and etoposide followed by autologous bone marrow rescue for the treatment of high risk central nervous system tumors. Bone marrow transplantation. 2000; 26:153160. [PubMed: 10918425]

23. Verschuur AC, Grill J, Lelouch-Tubiana A, Couanet D, Kalifa C, Vassal G. Temozolomide in paediatric high-grade glioma: a key for combination therapy? British journal of cancer. 2004; 91:425-429. DOI: 10.1038/sj.bjc.6601997 [PubMed: 15266331]

24. Jakacki RI, Cohen KJ, Buxton A, Krailo MD, Burger PC, Rosenblum MK, Brat DJ, Hamilton RL, Eckel SP, Zhou T, Lavey RS, Pollack IF. Phase 2 study of concurrent radiotherapy and temozolomide followed by temozolomide and lomustine in the treatment of children with highgrade glioma: a report of the Children's Oncology Group ACNS0423 study. Neuro Oncol. 2016; 18:1442-1450. DOI: 10.1093/neuonc/now038 [PubMed: 27006176]

25. Wolff JE, Wagner S, Reinert C, Gnekow A, Kortmann RD, Kuhl J, Van Gool SW. Maintenance treatment with interferon-gamma and low-dose cyclophosphamide for pediatric high-grade glioma. Journal of neuro-oncology. 2006; 79:315-321. DOI: 10.1007/s11060-006-9147-8 [PubMed: 16645718]

26. Wolff JE, Driever PH, Erdlenbruch B, Kortmann RD, Rutkowski S, Pietsch T, Parker C, Metz MW, Gnekow A, Kramm CM. Intensive chemotherapy improves survival in pediatric high-grade glioma after gross total resection: results of the HIT-GBM-C protocol. Cancer. 2010; 116:705-712. DOI: 10.1002/cncr.24730 [PubMed: 19957326]

27. Janssens GO, Gandola L, Bolle S, Mandeville H, Ramos-Albiac M, van Beek K, Benghiat H, Hoeben B, Morales La Madrid A, Kortmann RD, Hargrave D, Menten J, Pecori E, Biassoni V, von Bueren AO, van Vuurden DG, Massimino M, Sturm D, Peters M, Kramm CM. Survival benefit for patients with diffuse intrinsic pontine glioma (DIPG) undergoing reirradiation at first progression: A matched-cohort analysis on behalf of the SIOP-E-HGG/DIPG working group. European journal of cancer. 2017; 73:38-47. DOI: 10.1016/j.ejca.2016.12.007 [PubMed: 28161497]

28. Paganelli G, Grana C, Chinol M, Cremonesi M, De Cicco C, De Braud F, Robertson C, Zurrida S, Casadio C, Zoboli S, Siccardi AG, Veronesi U. Antibody-guided three-step therapy for high grade glioma with yttrium-90 biotin. European journal of nuclear medicine. 1999; 26:348-357.

[PubMed: 10199940] 


\section{Importance}

This work highlights a comprehensive systematic review and meta-analysis looking at outcomes of recurrent pediatric high-grade glioma spanning the last twenty years. To the authors' knowledge, this is the first meta-analysis reporting on outcomes of recurrent pediatric high-grade glioma. This work offers information on which future clinical trial design can be based and provides cumulative information on outcomes for providers, patients, and families. 


\section{References retrieved $\mathbf{n}=\mathbf{2 0 6 2}$}

MEDLINE 874; EMBASE 583; Cochrane 551; Web of Science 54

Duplicates removed $n=569$

\section{Reviewed for inclusion/exclusion criteria}

$$
n=1493
$$

$n=1467$

Age $n=45$

Diagnosis $n=641$

Recurrent $n=69$

Publication type $n=209$

Outcome $n=386$

Year $n=4$

Language $n=9$

Cannot determine age/diagnosis $\mathrm{n}=104$

Reviewed for statistical requirements for meta-analysis

No standard deviation $n=9$

Chemotherapy

$\mathrm{n}=11$

$\mathrm{n}=\mathbf{2 6}$

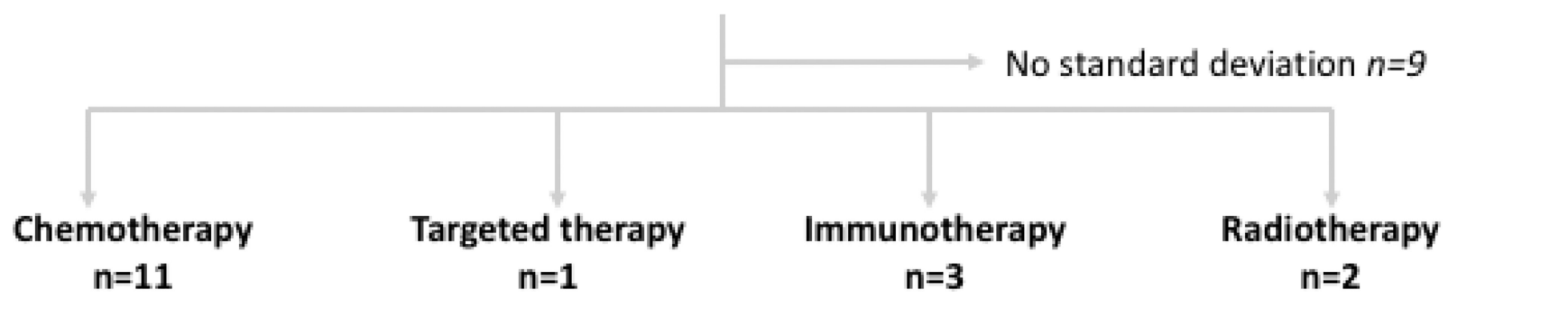

Fig 1.

Study inclusion/exclusion flowchart 
Study (Year)

Chamberlain (1997)

Papdakis (2000)

Wagner (2004)

Verschuur (2004)

De Sio (2006)

Shih (2008)

Parekh (2011)

Gilman (2011)

Ardon (2010)

Paganelli (1999)

Muller (2014)

Overall
PFS, months $(95 \% \mathrm{Cl})$

$4.0(-2.7,11)$

$3.5(1.7,5.3)$

$5.4(-4.8,16)$

$2.0(-7.4,11)$

$3.0(-2.7,8.7)$

$2.8(-4.1,9.7)$

$1.3(-18,20)$

$11(-18,39)$

$4.4(-15,24)$

$4.0(0.08,7.9)$

$8.0(-23,39)$

$3.5(2.1,5.0)$

Fig 2.

Forest plot representing PFS across all studies 
Chamberlain (1997)

Bouffet (1997)

Papdakis (2000)

Lashford (2002)

Wagner (2004)

De Sio (2006)

Shih (2008)

Gilheeney (2010)

Parekh (2011)

Gilman (2011)

Suarez (2009)

Ardon (2010)

Iwami (2012)

Lasky (2013)

Paganelli (1999)

Muller (2014)

Overall

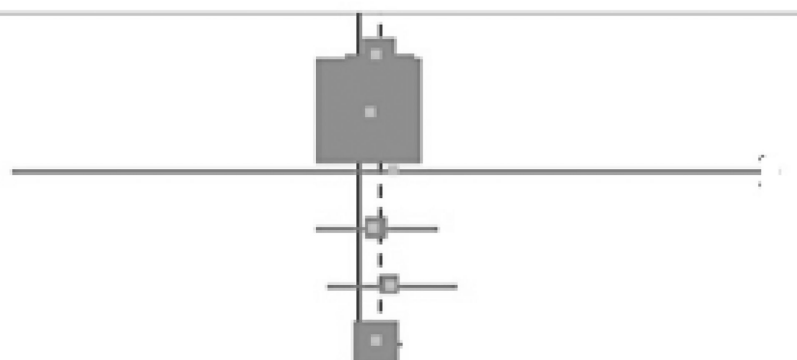

$5.5(-3.5,15)$

$3.5(1.1,5.9)$

$9.4(-91,110)$

$4.7(-11,21)$

$8.7(-8.4,26)$

$5.0(-1.3,11)$

$7.4(-88,103)$

$8.0(-77,93)$

$9.4(-8.2,27)$

$25(-17,67)$

$9.3(5.4,13)$

$13(-16,42)$

$6.5(-0.36,13)$

$7.0(-0.06,14)$

$15(1.9,28)$

$11(-11,34)$

$5.6(3.9,7.3)$

Fig 3.

Forest plot representing OS across all studies 


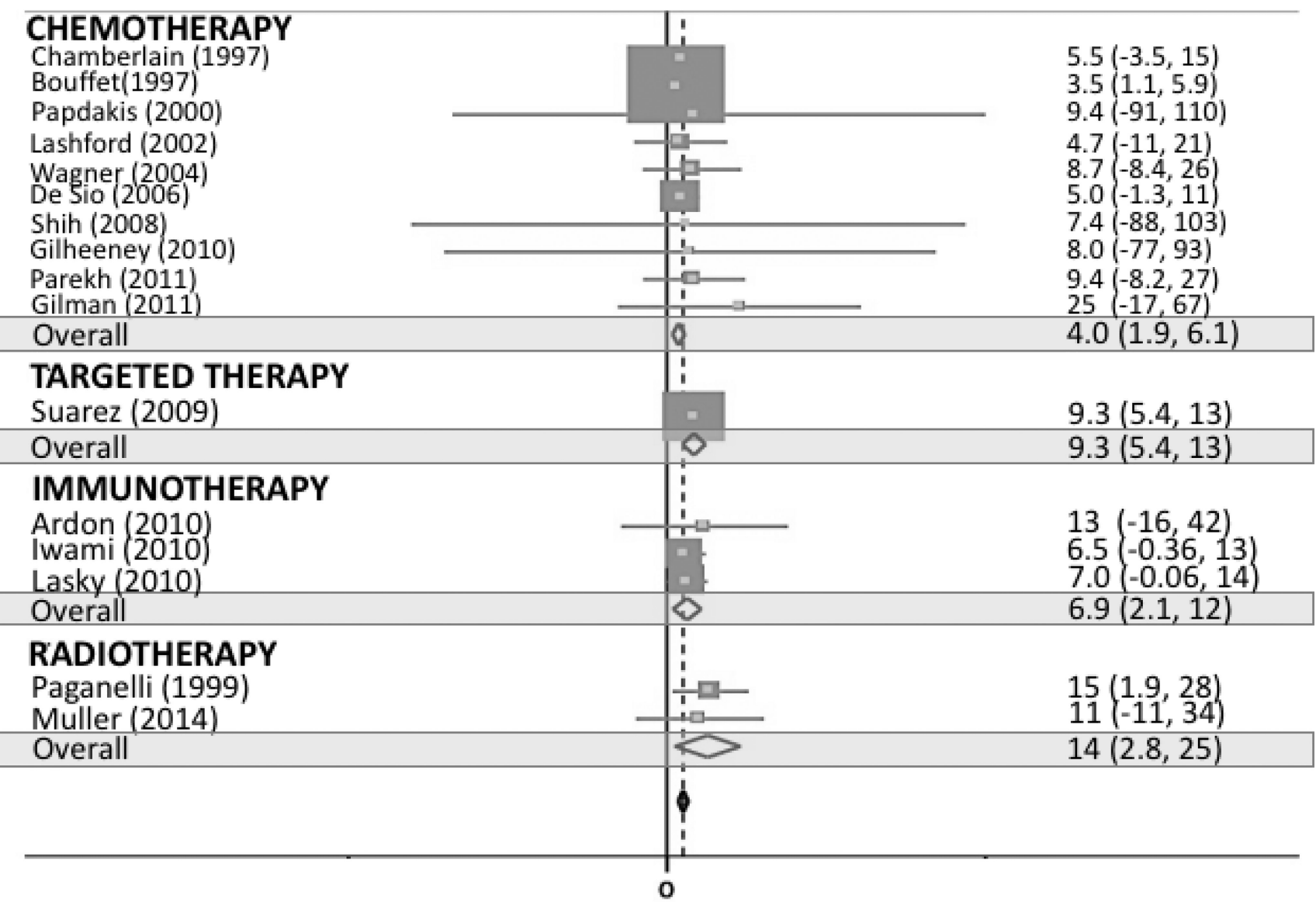

Fig 4.

Forest plot representing OS by therapy 
Study (Year) 1996-2006 Chamberlain (1997) Bouffet (1997)

Papdakis (2000) Lashford (2002)

Wagner (2004)

De Sio (2006)

Paganelli (1999)

Overall

2007-2016

Shih (2008)

Gilheeney (2010)

Parekh (2011)

Gilman (2011)

Suarez (2009)

Ardon (2010)

Iwami (2012)

Lasky (2013)

Muller (2014)
OS, months $(95 \% \mathrm{CI})$

$5.5(-3.5,15)$

$3.5(1.1,5.9)$

$9.4(-91,110)$

$4.7(-11,21)$

$8.7(-8.3,26)$

$5.0(-1.3,11)$ $15(1.9,28)$

$4.2(2.1,6.2)$

$7.4(-88,103)$

$8.0(-77,93)$

$9.4(-8.2,27)$

$25(-17,67)$

$9.3(5.4,13)$

$13(-16,42)$

$6.5(-0.36,13)$

$7.0(-0.06,14)$

$11(-11,34)$

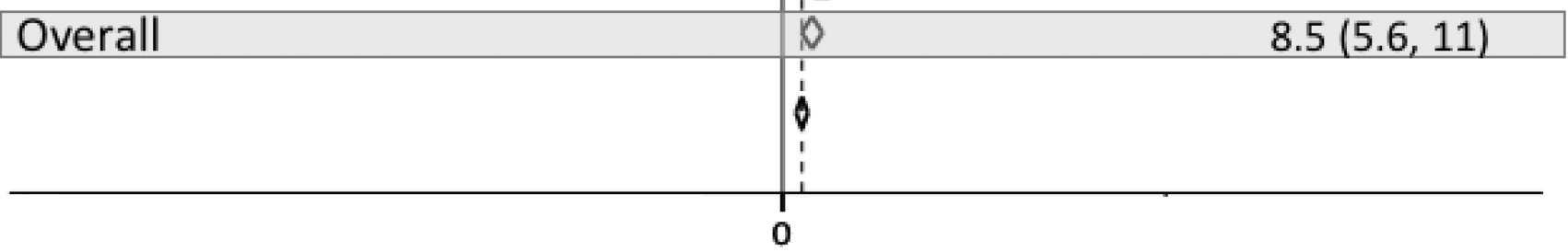

Fig 5.

Forest plot representing OS by decade 